\section{Magnetic reversal rate and sea level}

SIR-A negative correlation between the long-term eustatic sea level and the geomagnetic reversal rate for the past 150 million years (Myr) was recently analysed by Gaffin". We had previously pointed out the existence of such a correlation $^{2,3}$, although we did not do a complete mathematical analysis; nor did we detect the relatively small $10-\mathrm{Myr}$ phase shift reported by Gaffin. He attributes the correlation to long-term coupling between the flow pattern in the core (possibly responsible for spontaneous geomagnetic reversals) and the configuration of the mantle (which determines oceanic ridge volume and possibly sea level).

Our model presents a different explanation. We attribute a substantial fraction of the geomagnetic reversals to sudden changes in the moment of inertia of the Earth that occur during abrupt sea-level drops, when water is transferred from the oceans to continental ice at high latitudes; the faster rotation of the crust and mantle creates a velocity shear in the liquid core which disassembles the dynamo. A definite prediction of our theory is that the individual reversals (or geomagnetic excursions) should not lag the individual sea-level drops by more than a few times the characteristic convection time in the liquid core, a few thousand years. Our model successfully accounts for the initial sharp drop in the magnitude of the field at the beginning of a reversal and extended period of low field dominated by highorder multipole moments ${ }^{4,5}$, and also several previously unexplained correla- tions, including the association of geomagnetic reversals with microtektite layers ${ }^{6}$, craters $^{7}$, biological extinctions ${ }^{8}$ and short periods of cold climate ${ }^{9}$. The cooling that causes the abrupt sea-level drop could be induced by extraterrestrial impacts, volcanic eruptions or anything else that suddenly alters the global energy balance. Thus external events can change the flow pattern in the core, and induce geomagnetic reversals.

The negative correlation between the long-term eustatic sea level and the average rate of reversals is explained in our paper by the warmer Earth climate that existed during the period when the sea level was high, and the fact that larger (hence less frequent) cooling events were required to build up continental ice. A change in the rate of these events with time could give a phase shift such as that reported by Gaffin', although the individual reversals would continue to be coincident in time with individual cooling events.

R.A. Muller

D.E. MORRIS

Lawrence Berkeley Laboratory,

University of California,

Berkeley, California 94720, USA

1. Gaffin, S. Nature 329, 816-819 (1987).

2. Muller, R.A. \& Morris D.E. Geophys Res. Lett. 13, 1177 1180 (1986)

3. Schwarzchild, B. Physics Today 40, 17-20 (February 1987) 4. Jacobs, J.A. Reversals of the Earth's Magnetic Field, Ch.3 (Hilger, Bristol, 1984)

. Valet, J.P., Lal, C. \& Tucholka, P. Nature 322, 27 (1986).

6. Glass, B.P., Swincki, M.B. \& Zwart, P.A. Proc. lunar planet. Sci. Conf. 10, 2535-2545 (1979).

Pohl, J. Geologica bav, 75, 329-348 (1977)

8. Keany, J. \& Kennett, J.P. Deep Sea Res. 19, 529 (1972).

9. Krishnamurthy, R.V.et al. Nature 323, 150-152 (1986).

\title{
Punctuated equilibrium prevails
}

SIR-Sheldon's ${ }^{1}$ study of patterns of morphological change within eight lineages of mid-Ordovician trilobites provides additional evidence that palaeontological data are indeed relevant to contemporary evolutionary theory. Yet Maynard Smith's ${ }^{2}$ accompanying comment reveals an inaccurate grasp of the implications of Sheldon's study and the evolutionary issues that surround the idea of 'punctuated equilibria'. There is still a gap between the perception of evolutionary pattern and the efforts of theorists to understand causal relationships underlying evolutionary history.

Sheldon has studied changes in pygidial pleural rib counts in eight separate species-lineages of trilobites from the Builth inlier, spanning an interval of about 3 million years (Myr). With careful sampling, he has admirably taken advantage of the main strengths of the marine invertebrate fossil record. He has demonstrated within-sample variation in pygidial pleural rib counts, and has shown that modes may shift through time. We dispute his claim is to miss the larger point. Gingerich ${ }^{4}$ has presented patterns of size increase and decrease of various Eocene mammalian taxa forcefully reminiscent of Sheldon's trilobite patterns. Lande ${ }^{5}$ analysed some of Gingerich's data, concluding that the amount of change accrued over several million years of Eocene time was almost incomprehensibly neglible — far too slight to be distinguished from the effects of genetic drift, and too slight, perhaps, even to be comfortably explained as the consequence of natural selection. Most evolutionary biologists (including ourselves) see natural selection as a far more efficient causal agent of evolutionary change than Lande was able to demonstrate.

Much the same can be said of Sheldon's trilobites. A net increase of two or three pleural pygidial ribs over $3 \mathrm{Myr}$ in these trilobite lineages can hardly address the issues of patterns, and underlying processes, of changes that mark closely related, stratigraphically overlapping yet geographically disjunct congeneric species; and such small changes cannot have any real bearing on the origin of morphological differences that characterize taxa above the species level. The interplay of stochastic and deterministic processes tinkering with pygidial pleural rib numbers on the Builth trilobites for $3 \mathrm{Myr}$ yields little insight on the general dynamics of trilobite diversification. When taxa above the species level appear in the trilobite record, they do so rather abruptly. Evolutionary theorists must acknowledge ${ }^{6.7}$ that simple extrapolation of the amount of change that Sheldon documents over $3 \mathrm{Myr}$ would require vastly more than the 340-Myr recorded span of trilibite history to yield the morphological diversity attained within the Ordovician alone. Such rates of evolution are simply too slow to account for observed patterns of diversification.

Sheldon and Maynard Smith both think that the evidence of 'punctuation' resides in sudden, even quantal, morphological shifts wihin a stratigraphic succession of fossils from a single lineage. In contrast, we have always demanded stratigraphic overlap between two distinct taxa interpreted to be sister species, with the presumed ancestor persisting for some time alongside its putative descendant. The problem is to understand the origin of the consistent differences between the two species; only in such a context can (allopatric) speciation models be applied - the very heart and soul of punctuated equilibria.

Sheldon, citing Hughes's diagnoses ${ }^{8-10}$ of several non-stratigraphically overlapping species (chronospecies), and further citing his demonstration that Hughes's taxa are not discrete, but rather intergrade, concludes such a use of species names falsely gives the impression of punctuated 